\title{
Spatial Optimization and Mode Analysis of Primary Industry Structure in Yellow River Delta
}

\author{
Ping Yang ${ }^{1}$ and Yujian Yang ${ }^{2, *}$ \\ ${ }^{1}$ The institute of sustainable development of Shandong Agricultural Science Academy \\ ${ }^{2} \mathrm{~S} \& \mathrm{~T}$ Information Engineering Technology Center of Shandong Academy of Agricultural \\ Science, Information Center of Agronomy College of Shandong University \\ S\&T Information Engineering Research Center, Number 202 Gongye North Road, Licheng \\ District of Jinan, 250100, Shandong Province, P.R. China \\ yyjtshkh@gmail.com
}

\begin{abstract}
With the support of the total output of primary industry, secondary industry and tertiary industry and industry value of cultivating industry, forestry industry, animal husbandry industry and fishery industry of 19 country-level units in Yellow River Delta in 2007, the paper calculated the variation index of economic development based on country level, the MDS analysis was explored for primary industry, secondary industry and tertiary industry and compared with spatial difference characteristics of 19 country-level units in Yellow River Delta. The study carried out the analysis of combination analysis of primary, secondary and tertiary industry, and optimized the cultivating industry, forestry, animal husbandry and fishery industry. Autocorrelation characteristics was clarified by Moran`s I calculation, the new development mode of agricultural industrial structure was systemically concluded that the different mode presented in the different country units. The results showed that the industry evolution stage of primary, secondary and tertiary industry structure of 19 country units is in "secondary, primary and tertiary industries " stage and " secondary, primary and tertiary industries " stage according to industry structure stages theory, the obvious characteristics is that the cultivating industry value has spatial variability based on classical statistics combinig with universal Kriging. The emphasis of industry adjustment should be the focus on the secondary industry driving transformation into the primary, secondary and tertiary industries jointly driving economic growth, straight-pushing mode and tilting mode were put forward to new ideas about promoting industrial optimization and coordination development in Yellow River Delta. The study drew a conclusion that the measures were took to optimize industrial structure of cultivating industry, forestry industry, animal husbandry and fishery industry by combing straight-pushing mode with tilting mode, during the coordination development, focused on association effects of land and marine industry, extended agriculture industrialization chain and formed the diversification mode.
\end{abstract}

Keywords: Industry structure, Yellow-river Delta, Spatial analysis, Universal Kriging, New mode.

* Corresponding author.

D. Li and Y. Chen (Eds.): CCTA 2012, Part II, IFIP AICT 393, pp. 150-160, 2013.

(C) IFIP International Federation for Information Processing 2013 


\section{Introduction}

Industry structure was influenced by historical factors, natural factors and social\& economic factors, and its spatial heterogeneity is very close to the unbalance economic development. Differences of regional industrial structure which reflected the coordination of regional economic development is the result of long-term evolution a variety of factors, optimizing and upgrading of industry structure is helpful for the target of the regional economic development[1]. Accelerating transformation and adjustment of industry structure is important opportunity promoting development of rural areas and agriculture in Yellow River Delta, and adjustment of agriculture structure is also essential of rural areas and agriculture economic development.

The spatio-temporal model is important method for economic and social life study of the clear alternative to traditional analysis model. The spatial variability characteristics of industries value of primary, secondary and tertiary industry, and the cultivating industry, forestry industry, animal husbandry industry and fishery industry was explored and analyzed in Yellow River Delta in 2007, spatial difference of industry structure was developed to classify into different combination types, the continuity surface was used to establish model and prediction of the industries value. Further, the study provided the foundation for grasping the spatial difference of industry structure. On this basis, new mode was put forward to adjustment and upgrading of industry structure in Yellow River Delta.

\section{Material and Methodology}

\subsection{Overview in Yellow River Delta}

The yellow river delta was taken shape on the basis of the yellow river alluvial plain and the adjacent sea regions in the north parts of Shandong province, extension the round regions, including Dongying city, binzhou city, Hanting district, Shouguang city, Changyi city in Weifang city, Leling and Qingyun in Dezhou city, Gaoqing in Zibo city and Laizhou in Yantai city. The total area is $26500 \mathrm{Km} 2$, the $1 / 6$ of the total area in Shandong province. Speeding up the high-efficient and high-ecological development in yellow river delta, is not only promoting the regional economic development in Shandong, but also accelerating open-up of Binhai new district in Tianjin, at the same time, Establishment of ecological zone accelerating the development of Bohai economic zone and cultivating new economic growth pole in the lower reaches of the Yellow River. Some countries in Yellow River Delta are a crossing and overlay regions located in "high-efficient ecological zone in Yellow river delta and the blue economic zone of peninsula". It is crucial issue for adjustment and upgrading of industry structure during the constructing high-efficient ecological zone in Yellow river delta, not only beneficial from of the industry significance from macro perspective, but also engaging in high-efficiency, high-ecological and highindustrialization[2]. 


\subsection{Universal Kriging}

The Ji wheat 22 yield obtained from the actual yield of the observation area, with the Universal Kriging is a high precision prediction method one of Kriging methods, theoretical explanations of the method and technical aspects referred to the relevant literatures [3]. On the whole, this method not only removed the trend effect, but also has obvious characteristics of the residual prediction, the mathematical expectation of the regional variables was regarded as function of spatial position, effectively dealing with non-stationary, or drift characteristics of the regional variable. During the universal Kriging analysis. The general steps, firstly, analysis of data trends that exist in original data to the fit-goodness model. Secondly, the residual data (original data minus the trend data) was used to develop Kriging analysis. Finally, the comprehensive analysis of trend surface analysis and residual analysis of Kriging using the second-order trend surface equation, the final result is obtained by the separation of trend effects.

\section{$3 \quad$ Results and Analysis}

\subsection{Characteristics Analysis of Industry Structure in Yellow River Delta}

Variation index effectively reflected the difference of country-level economy in Yellow River Delta, as the following description: $S_{t}=\left[\sum\left(Y_{t i}-Y_{t}\right)^{2} / N\right]^{1 / 2} / Y_{t}$

Where, $N$ stands for country-level units number in Yellow River Delta, $Y_{t i}$ represents per GDP in the ith country in $t$ th, the larger variation index indicated the more difference of economic development in the regions[4,5].

As is illustrated in Figure 1, the variation index results of 19 country-level units in Yellow river delta referred to the following sequences: Kenli $>$ Guangrao $>$ Yangxin $>$ Zouping $>$ Dongying $>$ Leling $>$ Huimin $>$ Hekou $>$ Gaoqing $>$ Qingyun $>$ Laizhou $>$ Zhanhua $>$ Hanting $>\quad$ Bincheng $>$ Shouguang $>\quad$ Changyi $>$ Wudi $>$ Boxing $>$ Lijin,according to the sequence, the difference of economic development level presented smaller and smaller trend, the weak heterogeneity of economic development and the strong similarity, the difference of variation index reflected the spatial pattern of economic development and industry structure, the results indicated that there is balanced development characteristics, and is not in polarization situation of industry structure pattern in Kenli country, Guangrao country and Yangxin country. The relatively large heterogeneity difference of the regional economic developmen in Wudi Country, Boxing Country and Lijin Country. For the status, transformation and upgrading of industry structure should be utilized by the different policy and measures. 


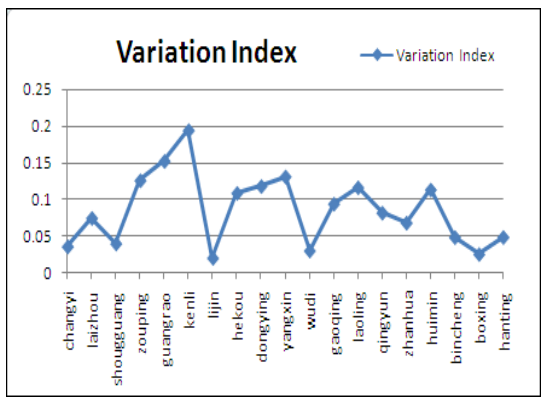

Fig. 1. The curve linear of 19 country units (left)

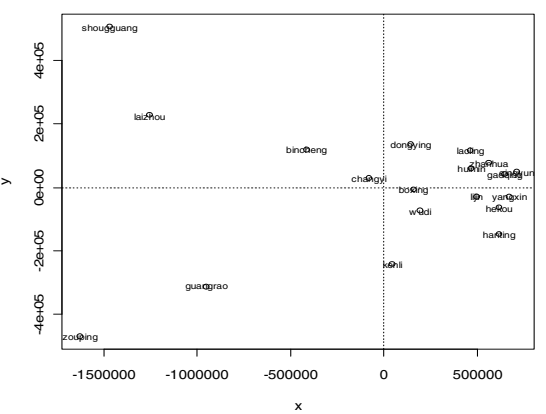

Fig. 2. The location plot based on multidimensional scaling analysis (right)

Multidimensional scaling method (multidimensional scaling, MDS) is a generic term that includes many different specific types, which can be classified according to the form of two-dimensional figure, its results was generally validated by the stress, the stress is less than 0.2 , which showed the reasonable MDS results, the stress is more than 0.05 , or equal to 0.05 and the stress is less than 0.1 or equal to 0.1 , the results showed that MDS results are considered of a good sequence, the stress is less than 0.05, with good representation.By the R system based on open-source software, the MDS value of primary industry, secondary industry, tertiary industry was calculated in Yellow River Delta, referred to figure 2. MDS can explain the coordination degree of industry structure and the similarity relationship of industry values[5,6].Generally, the total industry values and the coordination degree has the similarity characteristics located in the same quadrant or the near interval distance of country-level units, the results of MDS showed that there is similar to industry values mode about Shouguang and zouping, Guangrao and Laizhou,Wudi and Dongying,Laoling and Hanting and zhanhua, Boxing and wudi, Huimin gaoqing qingyun yangxin liji and hekou, Changyi and kenli has the special mode characteristics. In summary, the relationship between coordination goodness of industry structure and the total value of the primary, secondary industry and tertiary industry is very complex, the results also clearly showed that the goodness of industry structure is probably poor in the developed country units, but in the smaller total values country units, there is coordination goodness of industry structure. So industry restructuring should be in accordance with industry structure development and evolution in essence, and be treated differently.

In the study, the proportion of agricultural industrial structure and primary industry, secondary industry, tertiary industry was the following description in Yellow River Delta. In the definition the proportion of $0-25 \%$ is lower level structure, at the scope from $25 \%$ to $50 \%$ is defined as the lower level structure, the proportion scope of $50 \%-75 \%$ is defined as the high level, but the proportion of $75 \%-100 \%$ is regarded as the higher structure [7]. According to the above classification, the types combination of industry structure is classified into Figure 3, as follows: 
Low level +lower level+ lower level, the proportion of the primary industry is more than or equal to $0 \%$, less than $25 \%$; the proportion of the secondary industry is more than or equal to $25 \%$, less than $50 \%$; the proportion of the tertiary industry is more than or equal to $25 \%$, less than $50 \%$. The industry structure attributes to the type in Leling country, Qingyun country, Zhanhua country, the obvious characteristics is the lower value of the primary industry and the considerable proportion of the secondary industry and tertiary industry. The development emphasis in the next step is integration with city economy, increasing of the total economy of the secondary industry and tertiary industry.

Low level +higher level+ low level. The proportion of the primary industry is more than or equal to $0 \%$, less than $25 \%$; the proportion of the secondary industry is more than or equal to $25 \%$, less than $75 \%$; the proportion of the tertiary industry is more than or equal to $0 \%$, less than $25 \%$. The industry structure belongs to this type in Changyi country, Guangrao country, Lijin country, Hekou district, Yangxin country, Wudi country, Huimin country. The characteristics is the higher proportion of secondary industry, the lower proportion of primary industry and the tertiary industry, the development focus is on increasing proportion of tertiary industry based on industrial foundation.

Low level +higher level+ lower level. The proportion of the primary industry is more than or equal to $0 \%$, less than $25 \%$; the proportion of the secondary industry is more than or equal to $50 \%$, less than $75 \%$; the proportion of the tertiary industry is more than or equal to $25 \%$, less than $50 \%$. The industry structure belongs to this type in Laizhou city, Shouguang country, Dongying district, Gaoqing country, Bincheng district, Boxing, Hanting country. The characteristics is the higher proportion of the secondary industry and the tertiary industry, the lower proportion of the primary industry. The emphasis is increasing development of tertiary industry, strengthening the second industry and decreasing environmental damage.

Low level +high level+ lower level. The proportion of the primary industry is more than or equal to $0 \%$, less than $25 \%$; the proportion of the secondary industry is more than or equal to $75 \%$, less than $100 \%$; the proportion of the tertiary industry is more than or equal to $0 \%$, less than $25 \%$. The industry structure belongs to this type in Zouping country and Kenli country. The characteristics is the lower proportion of the primary industry and the tertiary industry, and the higher proportion of the secondary industry, the focus of development is upgrading the second industrial, accelerating the tertiary industry development.

During the evolution process of industry structure, adjustment and upgrading of it in developed regional showed the obvious evolution stages, " primary, secondary and tertiary industry " $\rightarrow$ " secondary, primary and tertiary industry " $\rightarrow$ " secondary, tertiary and primary industry " $\rightarrow$ " tertiary, secondary and primary industry ", the industry structure of "tertiary, secondary and primary industry" was regarded as the goodness level of industry structure. By calculation, there is " secondary, primary and tertiary industry " industry development stage in Lijin country, the industry evolution stage is " secondary, tertiary and primary industry " in Changyi city, Laizhou city, Shouguang country, Zouping city, Guangrao country, Kenli country, Hekou district, Dongying district, Yangxin country, Wudi country, Gaoqing country, Leling city, Qingyun 
country, Zhanhua country, Huimin country, Bincheng district, Boxing country, Hanting district. So the achievement of the superior level of industry structure was considered of the following country-level units: Laizhou city, Shouguang city, Dongying district, Gaoqing country, Leling city, Qingyun country, Zhanhua country, Bincheng district and Boxing country. In a word, three industry restructuring should depend on "high-efficient, ecological and economic zone plan in Yellow River Delta " and "the memorandum about joint promoting eco-agriculture demonstration zones in Yellow River Delta", the industry structure was carried out to mainly rely on the driving of secondary industry transformation into the collaboration driving of the primary industry, second industry and the tertiary industry, based on structural optimization and upgrading the joint development of the region and highlighting intensive utilization of resources, eco-efficient industrial system was preliminary constructed.

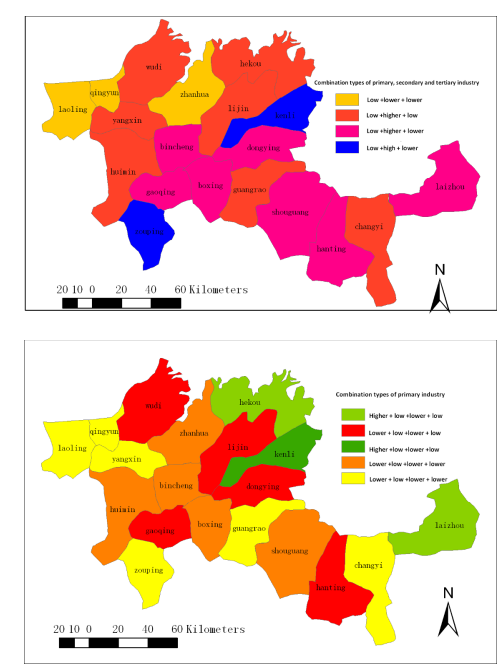

Fig. 3. Industry structure types of 19 country units in Yellow River Delta in 2007 (left)

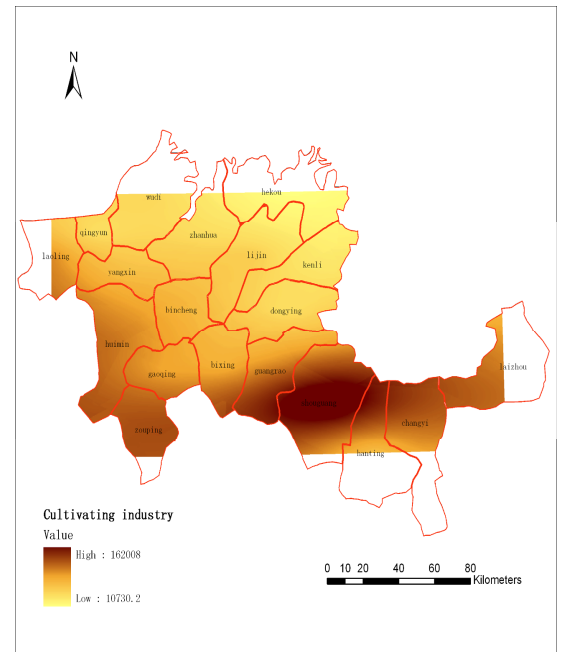

Fig. 4. The spatial prediction map of cultivating industry value of 19 country units in Yellow River Delta in 2007 (right)

\subsection{Optimization and Variability of Primary Industry Structure}

\subsubsection{Optimization Analysis of Primary Industry Structure}

"Agriculture, forestry, animal husbandry and fishery" industry is one of the 16 major industries reflecting the economic function [8]. The proportion of cultivating industry, forestry industry, animal husbandry and fishery industry value in Yellow River Delta showed that cultivating industry is the superior industry in Changyi city, Shouguang city, Zouping country, Guangrao country, Lijin country, Yangxin country, Gaoqing country, Leling, Qingyun country, Huimin, Bincheng district, Boxing country. Fishery industry is relatively prominent industry in Laizhou city, Kenli country and Hekou district. Industry structure types of cultivating industry, forestry industry, 
animal husbandry industry and fishery industry in Yellow River Delta is illustrated in Figure 3, as follows:

Higher level+ low level +lower level+ low level. Cultivating industry is prominent industry in Changyi, Zouping, Guangrao, Yangxin, Gaoqing, Leling, Qingyun, these country-level units are not only grain and cotton production base, but also the relatively good animal husbandry. Adjustment and upgrading of industry structure centered "Number 1" file in 2011, expanded the effective irrigation area, promoted high- efficient and high-quality of cultivating industry, moreover, the industry chain of vegetable, winter jujube was developed and extended, animal husbandry base was planned to construct, animal epidemic prevention system was built and the dairy chain was extended to construct the relatively reasonable economic structure.,

Lower level + low level +lower level+ low level. The industry structure belongs to the type in Dongying, Lijin, Wudi country, Hanting. For the four countries, Industrialization development should be added to the contents, which included "high technology, low resource consumption, little environmental pollution", so the new path of agriculture industrialization of information leading industrialization, industrialization promoting information was constructed to accelerate upgrading and adjustment of agricultural industry structure.

Higher level+low level+lower level+low level. Cultivating industry is prominent industry in Shouguang, Zhanhua, Huimin, Bincheng, Boxing. At the premise of ensuring the stable growth of grain, high quality and high efficient economic crops should be positive developed and fodder crops $s$ was gradually constructed to transform cultivating structure into ternary structure of grain crop, economic crop and fodder crop.

Lower level +low level +lower level+ lower level. High salinization content and fragile ecological environment is gradually improved in Kenli Country, with the comprehensive technology development of saline and alkaline land reclamation and water-saving demonstration development. In the next step, restructuring of industry structure should emphasize the coordinated development of agricultural industries centered industry effects of 1 adding $\mathrm{t} 1$ is more than 2 . For the new planning of the ecological function in the country, economic development and industry adjustment are the important thing, improving wetland ecological areas and cultivation of fishery industry, construction of aquaculture bases, the introduction of high-yield, highquality, high stress resistance breeding new varieties of sea water are the other emphasis in Yellow River Delta.

Lower level+ low level +lower level+ lower level. the kind of industry structure represented the good fishery foundation in Laizhou city and Hekou district, the aquaculture base, which is ecological health of the breeding base should be constructed in the country-level units, and the upgrading and restructuring of marine industries and traditional industries promoted the regional economic development.

There is obvious agglomeration characteristics of industries value of primary, secondary and tertiary industry, and the cultivating industry, forestry, animal husbandry and fishery industry, which suggested spatial association characteristics of industry values existed in 19 country-level units. Moran's I was used to analyze the cluster pattern of industries value. Theory and technology referred to the correlation 
literatures (Anselin,2004 ; YujianYang, 2010), Moran's I value is equal to 0.1034, 0.0484 and -0.0761 of primary industry value, secondary industry value and tertiary industry value, respectively, and Moran's I value is equal to $-0.0083,0.0989,0.2090$, 0.2202 and -0.0761 of primary industry value, secondary industry value and tertiary industry value, respectively. The biggest Moran's I value indicated the highest spatial autocorrelation characteristics, so the study results showed that animal husbandry value and the primary industry have the higher spatial autocorrelation, other industries has the lower autocorrelation characteristics, The autocorrelation characteristics of industries value was caused by the complicated factors, such as natural factors, social $\&$ economic factors and historical factors $[9,10]$.

\subsubsection{Spatial Variability of Cultivating Industry Value}

The classical statistics analysis was developed for the value of cultivating industry, forestry industry, animal husbandry industry and fishery industry of 19 country units in Yellow River Delta, the range reflected the regional difference of industry value, fishery industry value $>$ forestry industry value $>$ cultivating industry value $>$ animal husbandry industry value, and the variation coefficient is $0.657,1.028,0.807,0.537$, respectively, for cultivating industry value, fishery industry value, forestry value and animal husbandry value, the results showed that there existed in the big difference among industry values. The normality suggested that distribution of the above industry value is higher than normal distribution, the large standard deviation and coefficient of variation showed that relatively large differences existed in agriculture industrialization. The large skewness coefficient indicates the mean value of four industry value is the right side of the peak value, showing a positive (right) skewness, kurtosis coefficients is more than 0 showed that the distribution of four industry value is higher than normality, kurtosis coefficient of fishery industry and fishery industry value is more than 3 showed the fitting curve is steep.

The analysis of the Pearson's correlation coefficient among cultivating industry value, forestry industry value, animal husbandry value, and fishery industry value showed that the value is equal to 0.886 between cultivating industry value and animal husbandry value in confidence interval of $99 \%$, the value is equal to 0.532 between forestry industry value and fishery industry value in confidence interval of $95 \%$ and the value is equal to 0.463 between animal husbandry value and fishery industry value in confidence interval of $95 \%$. The striking result is that the better correlation characteristics among cultivating industry value, forestry industry value, animal husbandry value, and fishery industry value.

The spatial variability of industries value is not able to be clarified by the classical statistics, but using geostatistics combining with GIS. The continued surface of industry value using Universal Kriging referred to Figure 4. The results reflected the total distribution of cultivating industry value, based on the centroid coordinates of 19 country-level units, not only considering of sample size, shape, relationships and spatial location distribution, but also the non-stationary characteristics of industry value of samplings, centroid coordinates is calculated by the average weighted method and the formular is the following description: 


$$
X_{G}=\frac{\sum_{i=1}^{n} W_{i} X_{i}}{\sum_{i=1}^{n} W_{i}}, Y_{G}=\frac{\sum_{i=1}^{n} W_{i} Y_{i}}{\sum_{i=1}^{n} W_{i}}
$$

Where $X_{G}, Y_{G}$ represents the centroid coordinates; $X_{i}, \quad Y_{i}$ stands for the discrete coordinates of $i t h$ objective, $W_{i}$ represents the weight of $i t h$ objective.

\subsection{Mode Analysis of Primary Industry Structure}

There is a lack of proper balance and the low ratio among forestry industry, animal husbandry industry and fishery industry in Yellow River Delta and are still serious structural problems during the economy development. There are a lack of competitive industries and products, the low-quality varieties and the lag status of agricultural products processing and circulation, and so on. To solve these issues, we should improve the organization degree of agricultural industry, and build a new mode for regional industrial development [11].The new mode of the regional development combing straight-pushing mode with tilting mode was applied in the economy development in Yellow River Delta. Straight-pushing development mode emphasized the synchronous development of industries, or balanced development in the balanced regions of industry proportion, for example, Kenli country. Tilting development mode emphasized the development of the leading industry, which is the ideal regional model of industrial development comparative with straight-pushing development mode, the mode is suitable to Changyi city, Zouping country, Guangrao Country, Yangxin Country, Gaoqing Country, Laoling city and Qingyun country, dominant industry of cultivating industry. So tilting mode was also applied in the superior level of fishery industry in Laizhou, Kenli and Hekou[12].

We will continue to follow a new path of building industrial zone involved in marine industries and land industries, moreover, and we should accelerate the industry clustering concentrating on upgrading the industrial structure. The non-linear characteristics of 1 adding 1 is more than 2 from the association effects of industry, so we should emphasize the achievement development of industry clustering regions in Changyi, Shouguang, Laizhou, Wudi, Zhanhua and Bincheng district. With the support of economic technical development area, high-efficient and high-ecological agricultural demonstration zone, modern industrial clusters which has distinctive, specialized characteristics and complete service platform was constructed on basis of industry chain as a link, and industry demonstration area as the main object[13]. Agricultural industrial chain is loose, fragile in Yellow River Delta, and the regional chain is identical, short and narrow, the lack of diversity. Considering of it, the agricultural industrialization mode should combine with the service mode of agricultural information, such as "professional market (company) and farmers," "farmers and cooperative economic organization", "leading enterprise and farmers". The variety and quality of the agricultural products, the regional pattern of agriculture was carried out to adjust in all aspects, achieve the strategic adjustment and upgrading 
of agricultural structure, finally, the country industry model was formed, industry association characteristics of modern agriculture demonstration site of China of "Shouguang city mode" and industrial clusters of "Zouping city mode"[14,15].

\section{Conclusions}

In a word, the low level of industrial structure, the bigger proportion of traditional agriculture, the low-tech industry and the behind service industry were reflected in Yellow River Delta. There is the difference of country economy in 19 country-level units from variation index and multidimensional scaling index analysis. As is known to us, industrial structure types reflected the degree of industry optimization and provided the foundation of industry structure adjustment. By analyzing the combination types of industry structure of primary, secondary and tertiary industry, and the cultivating industry, forestry, animal husbandry and fishery industry of agricultural industrial structure, we drew a conclusion that the type of primary, secondary and tertiary industry structure of 19 country units located in Yellow River Delta is in the industrial structure "second, first and third" stage and "second, third and first" stage according to industry structure stages theory, which explained the necessity of upgrading and adjustment of industry structure. The results also showed that the value of cultivating industry has spatial variability based on classical statistics combining with universal Kriging, and the continued surface from point scale to the regional scale, the results also indicated that the optimization of industry structure in Yellow River Delta should take some important measures, such as the optimization of the agricultural industry chain and the boosting effects of the rural areas and agriculture information, the study also put forward to new ideas which straightpushing model is combing with favorable model of country economy about promoting industrial optimization and coordination development in Yellow River Delta. Correspondingly, we should accelerate efforts to upgrade the industrial structure and make independent innovations, and the emphasis of industry adjustment should be the focus on the secondary industry driving development transformation into the primary, secondary and tertiary industry jointly driving development, achievement of the transition from secondary industry serving as the major driving farce to primary, secondary and tertiary industries jointly driving economic growth, and accelerating the development of modern industrial system, integrating IT application with industrialization.

\section{References}

1. Cui, G., Wei, Q., Chen, Z.: Regional analysis and planning. High Education Press, Beijing (1999) (in Chinese)

2. Development and reform committee of Shandong province, the high-efficient and highecological economic zone in Yellow River Delta (2008)

3. Using ArcGIS Geostatistical Analyst. GIS by ESRI (2008) 
4. Wu, D.: The advanced tutorial of regional analysis and planning. High Education Press, Beijing (1999) (in Chinese)

5. Wang, B.: Multivariate statistical analysis and modeling of R language. Jinan University Press, Guangzhou (2010)

6. Li, W.: Application of multivariate statistical analysis. Beijing University Press, Beijing (2008)

7. Lijun: Geographical information and the multi-objective planning in the regional scale. Electronics Industry Press, Beijing (2006)

8. Wang, J., Lian, Y., Liuxin: Spatial ananlysis tutorial. Science Press, Beijing (2010)

9. Anselin, L., Florax, R.J.G.M., Rey, S.J.: Advances in spatial econometrics: methodology, tools and applications. Springer, Berlin (2004)

10. Yang, Y., Zhu, J., Wan, S., Zhang, X.: The spatial and temporal prognosis of oilseed yield in Shandong Province Springer. Computer and Computing Technologies in Agriculture, Daoliang li et al. pp. 146-157

11. http://www.moa.gov.cn/zwllm/zwdt/201008/ t20100803_1611790.html

12. Wang, C.: Collaboration development model of Country economic industry. China Economic Press, Beijing (2006)

13. Zhang, M.: Growth mechanism of agricultural industrialization chain. Science Press, Beijing (2010)

14. He, G., Wang, X.: Economic and industrial pattern of marine industry. Ocean Press, Beijing (2010)

15. Li, P., Wang, X.: Harmonious development — path of China count-level development. The People Press, Beijing (2010) 